Internet Engineering Task Force (IETF)

N. Hilliard

Request for Comments: 6666

Category: Informational

ISSN : $2070-1721$

INEX

D. Freedman

Claranet

August 2012

A Discard Prefix for IPv6

Abstract

Remote triggered black hole filtering describes a method of mitigating the effects of denial-of-service attacks by selectively discarding traffic based on source or destination address. Remote triggered black hole routing describes a method of selectively rerouting traffic into a sinkhole router (for further analysis) based on destination address. This document updates the "IPv6 Special Purpose Address Registry" by explaining why a unique IPv6 prefix should be formally assigned by IANA for the purpose of facilitating IPv6 remote triggered black hole filtering and routing.

Status of This Memo

This document is not an Internet Standards Track specification; it is published for informational purposes.

This document is a product of the Internet Engineering Task Force $(\mathrm{IETF})$. It represents the consensus of the IETF community. It has received public review and has been approved for publication by the Internet Engineering Steering Group (IESG). Not all documents approved by the IESG are a candidate for any level of Internet Standard; see section 2 of RFC 5741.

Information about the current status of this document, any errata, and how to provide feedback on it may be obtained at http://www.rfc-editor.org/info/rfc6666. 
Copyright Notice

Copyright (c) 2012 IETF Trust and the persons identified as the document authors. All rights reserved.

This document is subject to BCP 78 and the IETF Trust's Legal Provisions Relating to IETF Documents

(http://trustee.ietf.org/license-info) in effect on the date of publication of this document. Please review these documents carefully, as they describe your rights and restrictions with respect to this document. Code Components extracted from this document must include Simplified BSD License text as described in section $4 . e$ of the Trust Legal Provisions and are provided without warranty as described in the Simplified BSD License.

Table of Contents

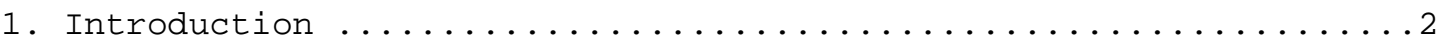

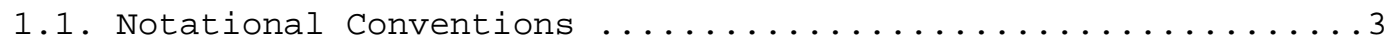

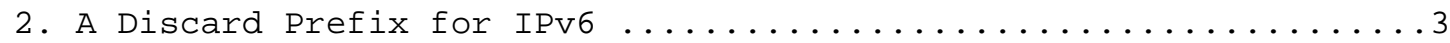

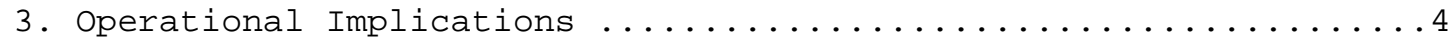

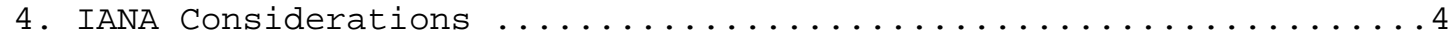

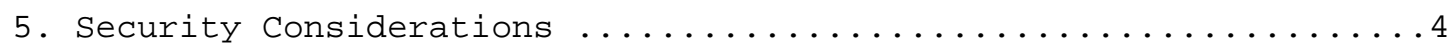

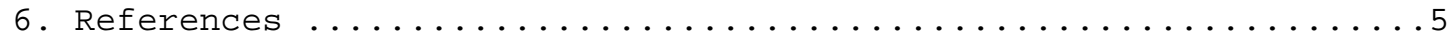

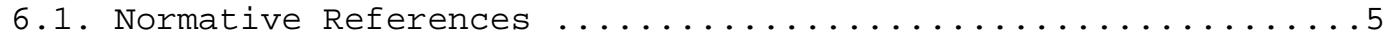

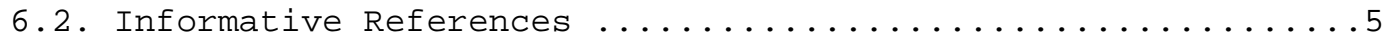

1. Introduction

Remote Triggered Black Hole (RTBH) filtering describes a class of methods of blocking IP traffic either from a specific source ([RFC5635]) or to a specific destination ([RFC3882]) on a network. RTBH routing describes a class of methods of re-routing IP traffic destined to the attacked/targeted host to a special path (tunnel) where a sniffer could capture the traffic for analysis. Both of these methods operate by setting the next-hop address of an IP packet with a specified source or destination address to be a unicast prefix that is connected locally or remotely to a router's discard, null, or tunnel interface. Typically, reachability information for this prefix is propagated throughout an autonomous system using a dynamic routing protocol such as BGP ([RFC3882]). By deploying RTBH systems across a network, traffic to or from specific destinations may be selectively black-holed or re-routed to a sinkhole device in a manner that is efficient, scalable, and straightforward to implement. 
On some networks, operators configure RTBH installations using [RFC1918] address space or the address blocks reserved for documentation in [RFC5737]. This approach is inadequate because RTBH configurations are not documentation, but rather operationally important features of many public-facing production networks. Furthermore, [RFC3849] specifies that the IPv6 documentation prefix should be filtered in both local and public contexts. On this basis, it is suggested that both private network address blocks and the documentation prefixes described in [RFC5737] are inappropriate for RTBH configurations and that a dedicated IPv6 prefix should be assigned instead.

This document updates the "IPv6 Special Purpose Address Registry" [IANA-IPV6REG].

\subsection{Notational Conventions}

The key words "MUST", "MUST NOT", "REQUIRED", "SHALL", "SHALL NOT", "SHOULD", "SHOULD NOT", "RECOMMENDED", "MAY", and "OPTIONAL" in this document are to be interpreted as described in [RFC2119].

\section{A Discard Prefix for IPv6}

For the purposes of implementing an IPv6 RTBH configuration, a unicast address block is required. There are currently no IPv6 unicast address blocks that are specifically nominated for the purposes of implementing such RTBH systems.

While it could be argued that there are other addresses and address prefixes that could be used for this purpose (e.g., documentation prefixes, private address space), or that an operator could assign an address block from their own address space for this purpose, there is currently no operational clarity on what address block would be appropriate or inappropriate to use for this purpose. By assigning a globally unique discard prefix for IPV6, the IETF will introduce good practice for the implementation of IPV6 RTBH configurations and will facilitate operational clarity by allowing operators to implement consistent and deterministic inter-domain prefix and traffic filtering policies for black-holed traffic.

As [RFC3882] and [RFC5635] describe situations where more than one discard address may be used for implementing multiple RTBH scenarios, a single address is not sufficient to cover all likely RTBH situations. Consequently, an address block is required. 


\section{Operational Implications}

This assignment MAY be carried in a dynamic routing protocol within an autonomous system. The assignment SHOULD NOT be announced to or accepted from third-party autonomous systems, and IPv6 traffic with a destination address within this prefix SHOULD NOT be forwarded to or accepted from third-party autonomous systems. If the prefix or a subnet of the prefix is inadvertently announced to or accepted from a third-party autonomous system, this may cause excessive volumes of traffic to pass unintentionally between the two networks, which would aggravate the effect of a denial-of-service attack.

On networks that implement IPv6 remote triggered black holes, some or all of this network block MAY be configured with a next-hop destination of a discard or null interface on any or all IPv6 routers within the autonomous system.

4. IANA Considerations

Per this document, IANA has recorded the allocation of the IPv6 address prefix 0100::/64 as a Discard-Only Prefix in the "Internet Protocol Version 6 Address Space" and added the prefix to the "IANA IPv6 Special Purpose Address Registry" [IANA-IPV6REG]. No end party has been assigned to this prefix. The prefix has been allocated from $:: / 3$.

5. Security Considerations

As the prefix specified in this document ought not normally be transmitted or accepted over inter-domain BGP sessions for the reasons described in section 3, it is usually appropriate to include this prefix in inter-domain BGP prefix filters [RFC3704] or otherwise ensure the prefix is neither transmitted to nor accepted from a third-party autonomous system. 
6. References

6.1. Normative References

[ IANA-IPV6REG]

Internet Assigned Numbers Authority, "IPv6 Special Purpose Address Registry", 2012, <http://www.iana.org/assignments/ iana-ipv6-special-registry>.

[RFC2119] Bradner, S., "Key words for use in RFCs to Indicate Requirement Levels", BCP 14, RFC 2119, March 1997.

[RFC3882] Turk, D., "Configuring BGP to Block Denial-of-Service Attacks", RFC 3882, September 2004 .

[RFC5635] Kumari, W. and D. MCPherson, "Remote Triggered Black Hole Filtering with Unicast Reverse Path Forwarding (uRPF)", RFC 5635, August 2009.

\subsection{Informative References}

[RFC1918] Rekhter, Y., Moskowitz, B., Karrenberg, D., de Groot, G., and E. Lear, "Address Allocation for Private Internets", BCP 5, RFC 1918, February 1996.

[RFC3704] Baker, F. and P. Savola, "Ingress Filtering for Multihomed Networks", BCP 84, RFC 3704, March 2004.

[RFC3849] Huston, G., Lord, A., and P. Smith, "IPv6 Address Prefix Reserved for Documentation", RFC 3849, July 2004.

[RFC5737] Arkko, J., Cotton, M., and L. Vegoda, "IPv4 Address Blocks Reserved for Documentation", RFC 5737, January 2010. 


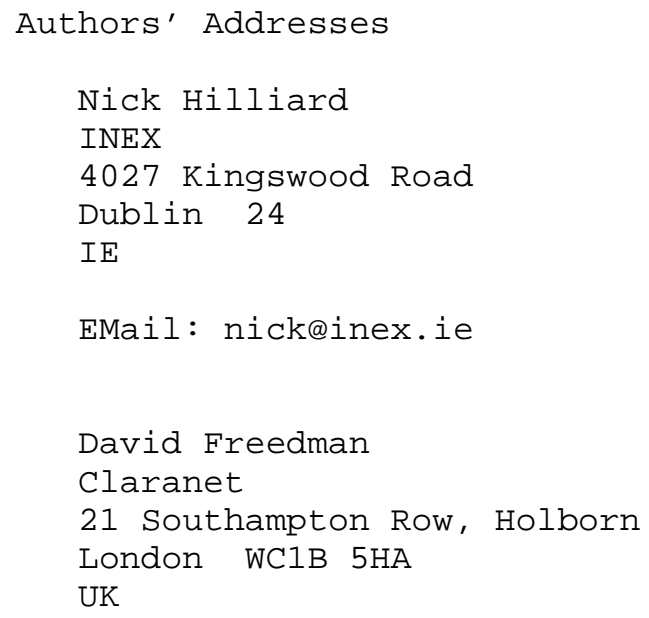

EMail: david.freedman@uk.clara.net 